\title{
A brief review of chronic obstructive pulmonary disease
}

\author{
James C Hogg MD PhD
}

\author{
JC Hogg. A brief review of chronic obstructive pulmonary disease. \\ Can Respir J 2012;19(6):381-384.
}

A recent study, based on a combination of multidetector computed tomography scanning of an intact specimen with microcomputed tomography and histological analysis of lung tissue samples, reported that the number of terminal bronchioles were reduced from approximately 44,500/lung pair in control (donor) lungs to approximately $4800 /$ lung pair in lungs donated by individuals with very severe (Global initiative for chronic Obstructive Lung Disease stage 4) chronic obstructive pulmonary disease (COPD) treated by lung transplantation. The present short review discusses the hypothesis that a rapid rate of terminal bronchiolar destruction causes the rapid decline in lung function leading to advanced COPD. With respect to why the terminal bronchioles are targeted for destruction, the postulated mechanisms of this destruction and the possibility that new treatments are able to either prevent or reverse the underlying cause of airway obstruction in COPD are addressed.

Key Words: Airway obstruction; Chronic airway inflammation; Emphysematous tissue destruction

The Global initiative for chronic Obstructive Lung Disease pulmonary disease (COPD) continues to increase in the developed world (1). Moreover, a recent worldwide population-based study indicating that its prevalence in the population $>40$ years of age is approximately $10 \%$ (2) suggests that COPD will remain a major worldwide public health problem until better methods of prevention and treatment are found. A diagnosis of COPD is made by measuring the volume of air that can be forcibly expired from total lung capacity in $1 \mathrm{~s}\left(\mathrm{FEV}_{1}\right)$ and comparing it with the forced vital capacity (FVC) measured during a similar manoeuvre when no time limit is applied. When the $\mathrm{FEV}_{1} / \mathrm{FVC}$ ratio is $\angle 70 \%$, COPD is said to be present and is assigned (Figure 1) to mild (GOLD 1), moderate (GOLD 2), severe (GOLD 3 ) and very severe (GOLD 4) categories based on the level of $\mathrm{FEV}_{1}$ expressed as a percent predicted. Figure 1 presents data collected by Fletcher and Peto (3) on the natural history of COPD. They measured $\mathrm{FEV}_{1}$ at different time points compared with the individuals's predicted $\mathrm{FEV}_{1}$ at 25 years of age. These data show the rate of decline that occurs in nonsmokers as they age compared with similar data on the majority of current smokers and those who stopped smoking over the same time period. Importantly, it shows that there is a susceptible minority of smokers and some nonsmokers who decline at a rate that will place them in the severe and very severe categories of COPD as they age. Some individuals may be in this category because poor lung development or childhood infection prevented them from reaching their predicted maximum at 25 years of age. The relative contribution of environmental exposure and genetic predisposition to those whose $\mathrm{FEV}_{1}$ declines rapidly remains poorly understood. Up until the 1960 s, the small conducting airways were believed to be the major site of obstruction to flow below the larynx in the normal lung. However, this opinion began to change with the appearance of Weibel's classic 1963 monograph on the anatomy of the human lung (4), which provided quantitative data showing that the total number and lumen crosssectional area of the small conducting airways increases exponentially

\section{Un bref examen de la maladie pulmonaire obstructive chronique}

Une récente étude, fondée sur une association de tomographie assistée par ordinateur à détecteurs multiples d'un spécimen intact et d'une tomographie assistée par ordinateur avec l'analyse histologique d'échantillons de tissus pulmonaires, a établi que le nombre de bronchioles terminales avait fléchi d'environ 44500 par paire de poumons chez les sujets témoins (donneurs) à environ 4800 par paire de poumons chez les personnes ayant une très grave maladie pulmonaire obstructive chronique (MPOC) (phase 4 selon la Global initiative for chronic Obstructive Lung Disease) traitée par greffe pulmonaire. La présente brève analyse porte sur l'hypothèse selon laquelle un taux rapide de destruction des bronchioles terminales provoque le rapide déclin de la fonction pulmonaire, menant à une MPOC avancée. Pour ce qui est des raisons pour lesquelles les bronchioles terminales sont ciblées, les chercheurs exposent les mécanismes postulés de leur destruction et la possibilité que de nouveaux traitements puissent prévenir ou renverser la cause sous-jacente de l'obstruction des voies aériennes en cas de MPOC.

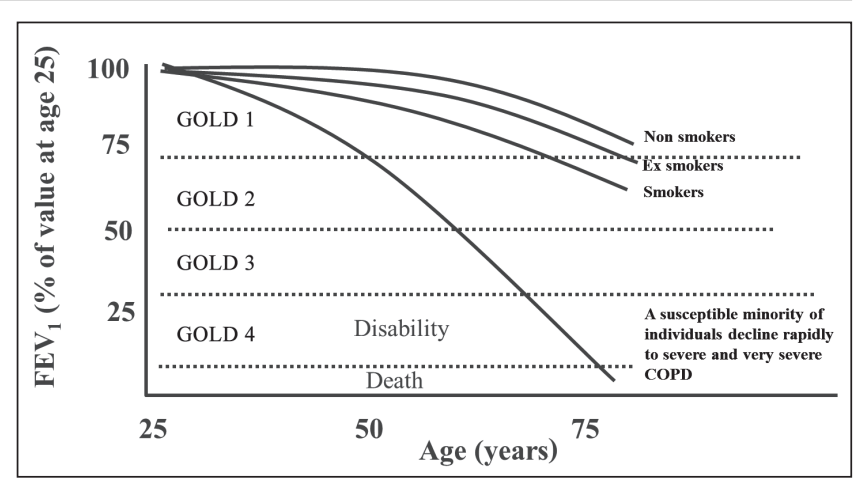

Figure 1) Data adapted from Fletcher and Peto (3) on the natural history of chronic obstructinve pulmonary disease (COPD) in relation to the four Global initiative for chronic Obstructive Lung Disease (GOLD) categories of COPD severity. It is postulated that the slow rates of decline in forced expiratory volume in $1 \mathrm{~s}\left(\mathrm{FEV}_{1}\right)$ observed in nonsmokers and in the majority of those who either smoke or have stopped smoking is caused by a slow rate of loss of terminal bronchioles. It is further postulated that the rapid decline in $\mathrm{FEV}_{1}$ observed in the susceptible minority and in those who fail to reach to reach the maximum expected $\mathrm{FEV}_{1}$ at 25 years of age is caused by a significantly more rapid rate of terminal bronchiolar loss in this group of individuals

with each generation of airway branching. This anatomical arrangement greatly improves the mixing of each tidal breath with the much larger volume of gas present in the lung at end expiration by slowing the bulk flow of inhaled gas and facilitating its diffusion into the distal airspaces. Green (5) subsequently used these new data to calculate the resistance to flow in the small airways and found that it was substantially less than the existing paradigm suggested. Shortly thereafter, Hogg et al (6) provided the first direct measurements of the resistance to

University of British Columbia Research Laboratories, St Paul's Hospital, Vancouver, British Columbia

Correspondence: Dr James C Hogg, University of British Columbia Research Laboratories, St Paul's Hospital, 1081 Burrard Street, Vancouver,

British Columbia V6Z 1Y6. E-mail jim.hogg@hli.ubc.ca 

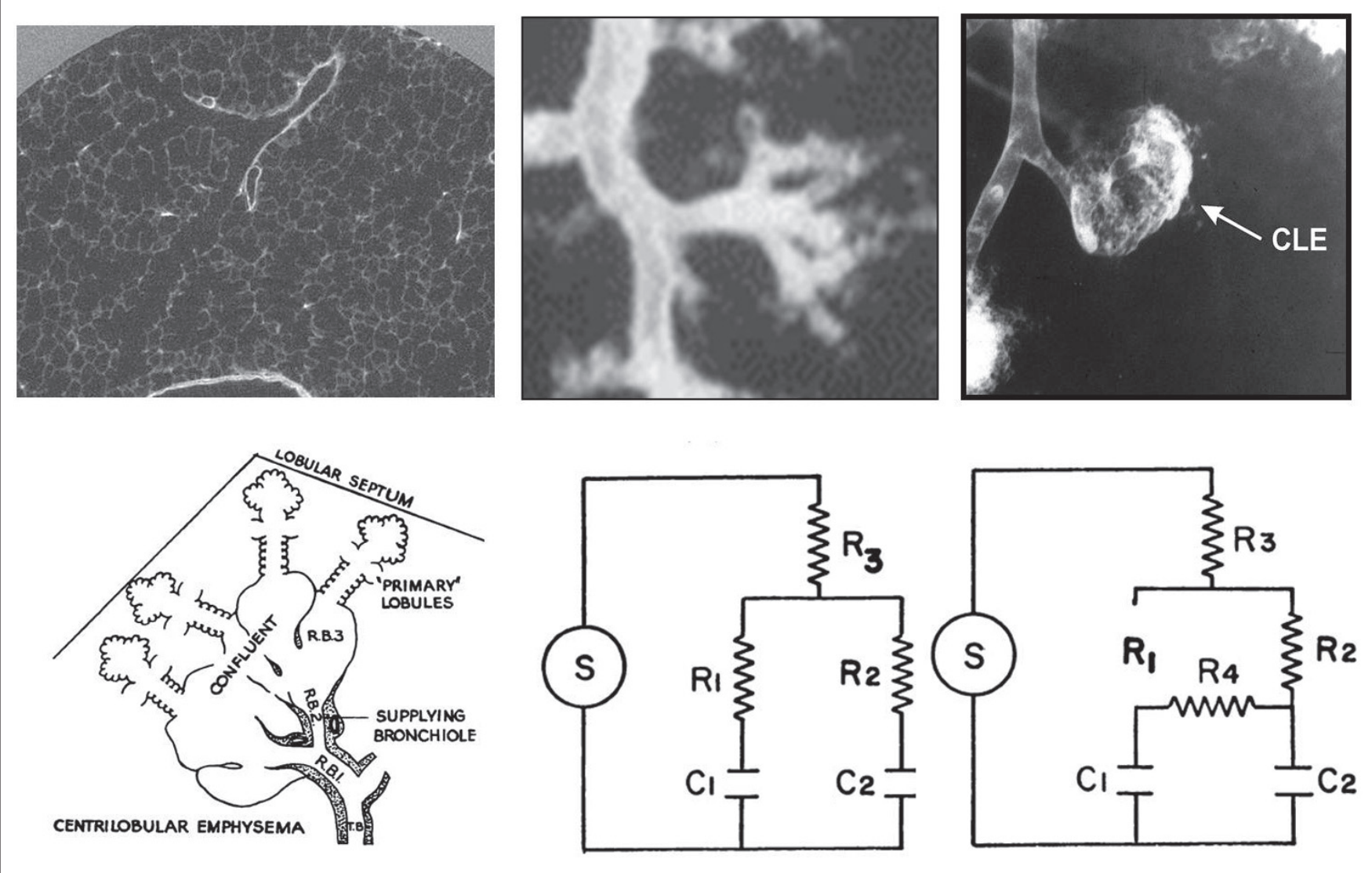

Figure 2) The top row of images left to right show a microcomputed tomography (microCT) image of a normal terminal bronchiole dividing into two respiratory bronchioles where alveoli first appear. A normal bronchogram in which a bronchiole divides into a cluster preterminal and terminal bronchioles to form a normal lung lobule (middle); and a centrilobular emphysematous (CLE) space formed by the coalescence of destroyed respiratory bronchioles supplied by several different terminal bronchioles (right). The bottom row of images left to right show a diagram of Leopold and Gough's (12) concept of a primary centrilobular lesion in which thick-walled narrowed terminal bronchioles (TB) supply destroyed respiratory bronchioles (RB). A circuit diagram (bottom center) of normal lung anatomy in which preterminal bronchioles (R3) supply terminal bronchioles R1 and R2 that supply alveoli labelled C1 and C2. As well as a second circuit diagram (bottom right) showing the abnormal situation in CLE in which destruction of a terminal bronchiole indicated by removal of $R 1$ leave isolated normal alveoli $\mathrm{C} 1$ to be supplied by the enlarged low resistance collateral channels $\mathrm{R} 4$ opened by centrilobular emphysematous destruction that develops beyond thickwalled terminal bronchioles that survive the destructive process

flow in human airways $<2 \mathrm{~mm}$ in diameter and established that these airways accounted for only approximately $20 \%$ of the total resistance below the larynx but are the the major site of airway obstruction in COPD. These results have been confirmed by others (7). This increase in resistance to flow in the small conducting airways combined with emphysematous destruction of the elastic recoil force available to drive air out of the lung accounts for the reduction in $\mathrm{FEV}_{1}$ and $\mathrm{FEV}_{1} / \mathrm{FVC}$ in COPD. Moreover, because the product of resistance $\left(\mathrm{cmH}_{2} \mathrm{O} / \mathrm{L} / \mathrm{s}\right)$ and lung elastic recoil $\left(\mathrm{L} / \mathrm{cmH}_{2} \mathrm{O}\right)$ has the units of time, their combined effect determines the time taken for the lung to empty during forced expiration (8). Therefore, individuals with mild disease who experience no breathing discomfort may develop symptoms of dyspnea and chest tightness during exercise because the increased rate of breathing provides too little time for the lung to return to the normal end expiratory volume at the end of each breath (9).

The boundary region between purely conducting airways and the gas-exchanging surface of the lung is formed by the division of terminal bronchioles into first-order respiratory bronchioles where alveoli first appear. The top left image in Figure 2 shows a microcomputed tomography (microCT) image of a terminal bronchiole dividing into the two proximal respiratory bronchioles it supplies within a single acinus. The top middle figure shows a bronchogram of the cluster of preterminal and terminal bronchioles only millimeteres apart in a normal lung lobule and the top right image shows a cetrilobular lesion formed by the coalescence of several destroyed acini within a single lobule. A recent quantitative microCT and multidetector computed tomography study of donor lungs (10) showed that there are approximately a mean $( \pm$ SD) of $44,510 \pm 15,574$ terminal bronchioles per lung pair and this value compares favourably with the mean of $44,500 \pm 18,574$ terminal bronchioles calculated from four previously published studies of human lung casts. Surprisingly, the same study showed that, in persons who experienced rapid decline in $\mathrm{FEV}_{1}$ to end-stage disease and were treated by lung transplantation, this number of terminal bronchioles is reduced to approximately $10 \%$ of the control value in the centriloblar and approximately $25 \%$ of this control value in the panlobular emphysematous phenotypes of very severe (ie, GOLD 4) COPD (10). Because this destruction of terminal bronchioles occurred before the onset of emphysematous destruction, it is postulated that it begins early and accounts for the slow decline in $\mathrm{FEV}_{1}$ in nonsmokers and most smokers as they age. Moreover, it is further postulated that a more rapid rate of terminal bronchiolar destruction accounts for the rapid decline in $\mathrm{FEV}_{1}$ that leads to severe and very severe disease in the susceptible minority of smokers who develop COPD. Although this hypothesis requires additional work on persons with mild disease, it also raises important questions including the following: why are small conducting airways targeted for early destruction in COPD?; what is the mechanism(s) responsible for this destruction?; what is the time course over which 
this reduction in terminal bronchioles occurs?; and what is the relationship between terminal bronchiolar and emphysematous destruction of gas-exchanging tissue?

The major risk factor for COPD is the persistent inhalation of toxic particles and gases caused primarily, but not exclusively, by smoking tobacco (1). Other similar risk factors include the polluted personal atmosphere surrounding those who cook food in much of the developing world, chronic exposure to exhaust from internal combustion engines and a variety of other industrial and domestic processes that contaminate the atmosphere with toxic airborne particles (1). Previous work has shown that airborne particles $>0.5 \mu \mathrm{m}$ in diameter tend to leave the stream of flowing gas and impact the walls of larger airways as the gas flow changes direction at airway branch points. In contrast, airborne particles $<0.5 \mu \mathrm{m}$ in diameter are capable of reaching the region of the airways where the flow slows and diffusion of gas begins. However, because particles $<0.5 \mu \mathrm{m}$ in diameter are approximately 1000 times larger than oxygen molecules and have a root mean square diffusion capability of only $10 \mu \mathrm{m} / \mathrm{s}$ compared with $6 \mathrm{~mm} / \mathrm{s}$ (ie, $6000 \mu \mathrm{m} / \mathrm{s}$ ) for molecules the size of oxygen (11), these small particles are significantly more likely to deposit in the transitional region of the airways because they are unable to diffuse deeper into the alveolar air spaces. In addition, the fact that the surface area of terminal bronchioles is much smaller than respiratory bronchioles where alveoli first appear means that the dose of particles per unit surface area is greater in terminal than in respiratory bronchioles. Therefore, it is reasonable to postulate that terminal bronchioles are destroyed before the respiratory bronchioles because they receive a higher dose of particulates. Images in Figure 2 show the consequences of this destruction. The figure on the bottom left shows Leopold and Gough's interpretation of the primary centrilobular lesion (12). The images in the lower centre and right of Figure 2 represent electrical circuit models of the normal anatomy in which a preterminal bronchiole divides into two terminal bronchioles (bottom centre) and centrilobar emphysema (bottom right) in which destruction of one terminal bronchiole isolates normal alveoli that are supplied by enlarged collateral channels formed by emphysematous destruction beyond the remaining terminal bronchiole.

The mechanism(s) of lung tissue destruction in COPD is linked to the tissue repair process stimulated by repetitive inhalational injury. This type of tissue repair differs fundamentally from the tissue repair process that follows a single injury. Although both can activate the coagulation cascade to initiate damage control and stimulate an inflammatory response to both protect the tissue from infection and initiate the demolition of dead and dying tissue, the major difference is that following a single injury, the inflammatory process recedes as fibroblasts migrate into the tissue to lay down matrix that eventually form a permanent scar, whereas repetitive injury is associated with a persistent inflammatory process, tissue destruction and abnormal scar formation (ie, fibrosis) (13-15). The proteolysis/antiproteolysis imbalance theory for emphysematous tissue destruction in COPD was derived from observations that linked low circulating levels of alpha 1 -antitrypsin (A1AT) to the presence of panlobular emphysematous destruction (16). The theory was supported by experimental evidence showing that A1AT was a major inhibitor of neutrophil elastase and that instillation of neutrophil elastase into animal lungs produced emphysema-like lesions (17). This hypothesis was subsequently extended to tobacco smoke-induced emphysematous destruction by experiments showing that serum elastase inhibiting capacity was suppressed by exposure to tobacco smoke and that this suppression could be prevented by adding antioxidants to the serum (18). Further experiments demonstrating that the oxidation of methionine at either position 351 or 358 of A1AT suppressed its elastase binding capability provided a molecular basis for the integrating of oxidant/antioxidant mechanisms with the proteolysis/antiproteolysis theory (19). Additional application of molecular techniques to this problem revealed that the transcription factor $\mathrm{Nrf} 2$, responsible for regulating the cytoprotective and detoxifying programs that prevent toxic oxygen damage, is downregulated in lung tissue from persons with COPD $(20,21)$. Very recent experiments have shown that the phytochemical compound sulforaphane can elevate Nrf2, improve bacterial recognition and phagocytosis of human alveolar macrophages in patients with COPD, and improve bacterial clearance and killing in wild-type but not in Nrf2-deficient mice (22). Collectively, these results support the hypothesis that improved antioxidant therapy might prevent tissue destruction in COPD; however, this hypothesis has not been tested in clinical trials.

Studies of the repair process indicate that fibroblasts isolated from lungs affected by COPD behave differently than normal lung fibroblasts $(23,24)$. In addition, studies of an animal model of Marfan syndrome have established that the emphysematous destruction associated with this syndrome can be prevented in a mouse model of Marfan syndrome by either blocking the transforming growth factor-beta pathway with a neutralizing antibody or administering the angiotensin receptor II blocker losartan (25). More importantly, a second report from the same group has shown that losartan can also reverse tobacco smoke-induced emphysema in wild-type mice $(26,27)$. These observations suggest that angiotensin receptor blockers are able to slow the rate of tissue destruction in COPD. Other intracellular signalling pathways known to be involved in tissue repair that have also been implicated in the pathogenesis of COPD include the vascular endothelial growth factor (VEGF), Wnt and iNos signalling pathways. For example, the chronic blockade of the VEGF signalling pathway critical to angiogenesis and the formation of new vessels during tissue repair produces emphysema-like lesions in the lungs of rats (28). In addition, the Wnt signalling pathways that are known to participate in the control of inflammation, programmed cell death and mobilization of stem cell reservoirs within damaged tissue (29), have recently been implicated in emphysematous destruction by experiments showing that stimulation of Wnt signalling by lithium chloride reverses tobacco smoke-induced emphysema in smoke-exposed mice (30). Furthermore, mice lacking inducible nitric oxide synthase have been shown to be protected against tobacco smoke-induced emphysema, and treatment of wild-type mice with an inducible nitric oxide synthase inhibitor both prevented and reversed tobacco smoke-induced emphysematous destruction in mice (31). Although these animal studies are interesting, no clinical trials involving patients with COPD have been reported.

\section{SUMMARY}

The hypothesis that terminal bronchioles are the primary target for tissue destruction in COPD is supported by microCT evidence that destruction of the terminal bronchioles precedes emphysematous destruction. This observation is attributable to the increased dose of toxic particulates delivered to these airways because the smaller particles that reach the transitional region of the airways tend to remain there, being unable to diffuse more deeply into the lungs. The observation that terminal bronchiolar destruction isolates alveoli supplied by the destroyed airways, coupled with the observation that the centrilobular spaces are always supplied by patent terminal bronchioles, strongly supports the hypothesis that centrilobular emphysematous destruction develops beyond surviving terminal bronchioles and supplies isolated alveoli with collateral ventilation. Although studies of the molecular mechanisms of tissue destruction in animal models of tobacco smoke-induced emphysema are beginning to provide clearer insight into the pathogenesis, the mechanism of tissue destruction appears to be significantly more complex than anticipated, and it is possible that different mechanisms produce similar pathological results. Nevertheless, these new results provide hope that therapeutic agents capable of either preventing or reversing the rapid decline in $\mathrm{FEV}_{1}$ that leads to the dreadful consequences of end-stage COPD will eventually emerge. 


\section{REFERENCES}

1. Global Initiative for Chronic Obstructive Lung Disease (GOLD). Global strategy for the diagnosis, management, and prevention of chronic obstructive pulmonary disease (updated 2009) $<$ www.goldcopd.org > (Accessed May 31, 2012).

2. Buist AS, McBurnie MA, Vollmer WM, et al; Bold study of the prevalence of obstructive lung disease International variation in the prevalence of COPD (the BOLD Study): A population-based prevalence study. Lancet 2007;370:741-50.

3. Fletcher C, Peto R. The natural history of chronic airflow obstruction. BMJ 1977;1:1645-81.

4. Weibel ER. Morphometry of the Human Lung. New York: Academic Press Inc; 1963.

5. Green M. How big are the bronchioles? St Thomas Hospital Gazette. 1965;63:136-9.

6. Hogg JC, Macklem PT, Thurlbeck WM. Site and nature of airway obstruction in chronic obstructive lung disease. N Engl J Med 1968;1355-60.

7. Yanai M, Sekizawa K, Ohrui T, Sasaki H, Takishima T. Site of airway obstruction in pulmonary disease: Direct measurement of intrabronchial pressure. J Appl Physiol 1992;72:1016-23.

8. Mead J, Turner JM, Macklem PT, Little JB. Significance of the relationship between lung recoil and maximum expiratory flow. J Appl Physiol 1967;22:95-108.

9. Guenette JA, Webb KA, O’Donnell DE. Does dynamic hyperinflation contribute to dyspnoea during exercise in patients with COPD? Eur Respir J 2012;40:322-9.

10. McDonough JE, Yuan R, Suzuki M, et al. Small-airway obstruction and emphysema in chronic obstructive pulmonary disease. N Engl J Med 2011;365:1567-75.

11. Altschuler B, Yarmus L, Palmes ED, Nelson N. Aerosol deposition in the human respiratory tract. AMA Arch Ind Health 1957;15:292-303.

12. Leopold JG, Gough J. The centrilobular form of hypertrophic emphysema and its relation to chronic bronchitis. Thorax 1957;219-35.

13. Wynn TA, Ramalingam TR. Mechamisms of fibrosis: Therapeutic translation for fibrotic disease. Nat Med 2012;18:1028-40.

14. Kumar V, Abbas AK, Fausto N. Tissue renewal and repair. In: Robbins and Cotran pathologic basis of disease, 7 th edn. Philadelphia: Elsevier Saunders; 2004:87-118.

15. Madden JW, Peacock EE Jr. Studies on the biology of collagen during wound healing. 3. Dynamic metabolism of scar collagen and remodeling of dermal wounds. Ann Surg 1971;174:511-20.

16. Laurell CB, Erikssin S. The electrophoretic alpha 1-globulin pattern of serum in alpha 1-antitripsin deficiency. Scand J Clin Invest 1963;15:132-40.

17. Kaplan PD, Kuhn C, Pierce JA. The induction of emphysema with elastase. I. The evolution of the lesion and the influence of serum. J Lab Clin Med 1973;82:349-56.
18. Carp H, Janoff A. Possible mechanisms of emphysema in smokers. In vitro suppression of serum elastase-inhibitory capacity by fresh cigarette smoke and its prevention by antioxidants. Am Rev Respir Dis 1978;118:617-21.

19. Taggart C, Cervantes-Laurean D, Kim G, et al. Oxidation of either methionine 351 or methionine 358 in alpha 1 -antitrypsin causes loss of anti-neutrophil elastase activity. J Biol Chem 2000;275:27258-65.

20. Suzuki M, Betsuyaku T, Ito Y, et al. Down-regulated NF-E2-related factor 2 in pulmonary macrophages of aged smokers and patients with chronic obstructive pulmonary disease. Am J Respir Cell Mol Biol 2008;39:673-82.

21. Malhotra D, Thimmulappa R, Navas-Acien A, et al. Decline in NRF2-regulated antioxidants in chronic obstructive pulmonary disease lungs due to loss of its positive regulator, DJ-1. Am J Respir Crit Care Med 2008;178:592-604.

22. Harvey CJ, Thimmulappa RK, Sethi S, et al. Targeting Nrf2 signaling improves bacterial clearance by alveolar macrophages in patients with COPD and in a mouse model. Sci Transl Med 2011;3:78ra32.

23. Togo S, Holz O, Liu X, et al. Lung fibroblast repair functions in patients with chronic obstructive pulmonary disease are altered by multiple mechanisms. Am J Respir Crit Care Med 2008; 178:248-60.

24. Zandvoort A, Postma DS, Jonker MR, Noordhoek JA, Vos JT, Timens W. Smad gene expression in pulmonary fibroblasts: Indications for defective ECM repair in COPD. Respir Res 2008;9:83.

25. Habashi JP, Judge DP, Holm TM, et al. Losartan, an AT1 antagonist, prevents aortic aneurysm in a mouse model of Marfan syndrome. Science 2006;312:117-21.

26. Podowski M, Calvi C, Metzger S, et al. Angiotensin receptor blockade attenuates cigarette smoke-induced lung injury and rescues lung architecture in mice. J Clin Invest 2012;122:229-40.

27. Neptune ER, Frischmeyer PA, Arking DE, et al. Dysregulation of TGF-beta activation contributes to pathogenesis in Marfan syndrome. Nat Genet 2003;33:407-411.

28. Kasahara Y, Tuder RM, Taraseviciene-Stewart L, et al. Inhibition of VEGF receptors causes lung cell apoptosis and emphysema. J Clin Invest 2000;106:1311-9.

29. Whyte JL, Smith AA, Helms JA. Wnt signaling and injury repair. Cold Spring Harb Perspect Biol 2012;4:a008078.

30. Kneidinger N, Yildirim AÖ, Callegari J, et al. Activation of the WNT/B-catenin pathway attenuates experimental emphysema. Am J Respir Crit Care Med 2011;183:723-33.

31. Seimetz M, Parajuli N, Pichl A, et al. Inducible NOS inhibition reverses tobacco-smoke-induced emphysema and pulmonary hypertension in mice. Cell 2011;147:293-05. 


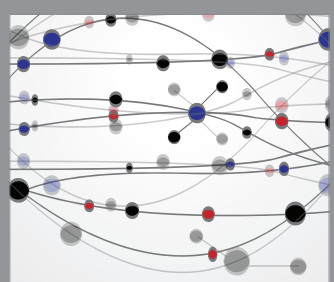

The Scientific World Journal
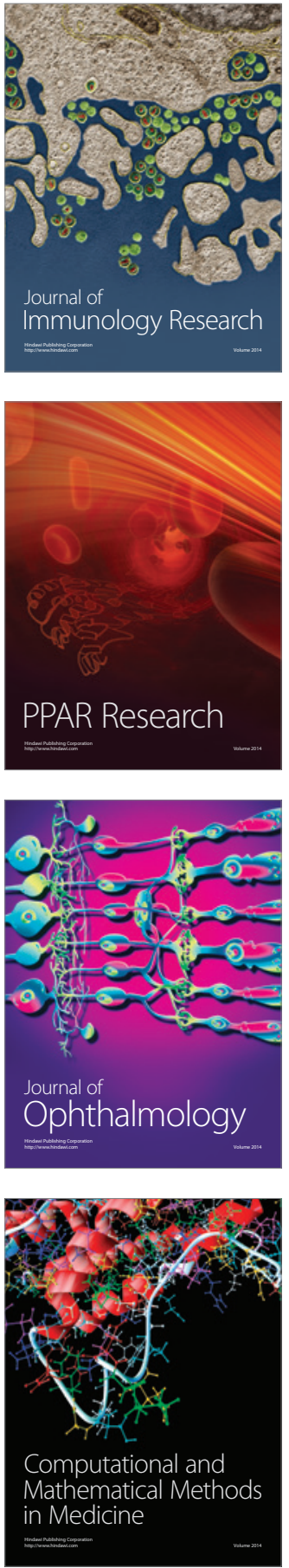

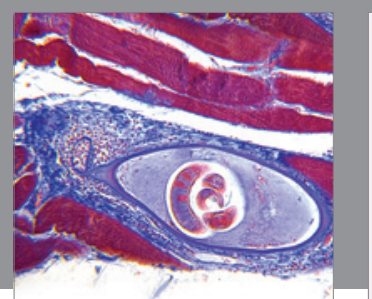

Gastroenterology Research and Practice

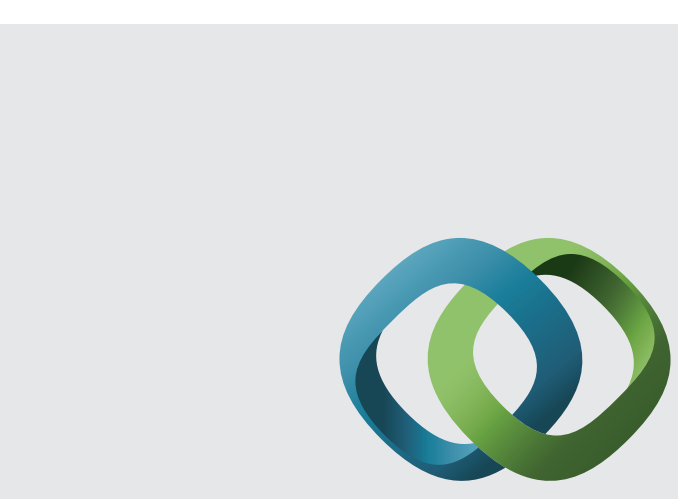

\section{Hindawi}

Submit your manuscripts at

http://www.hindawi.com
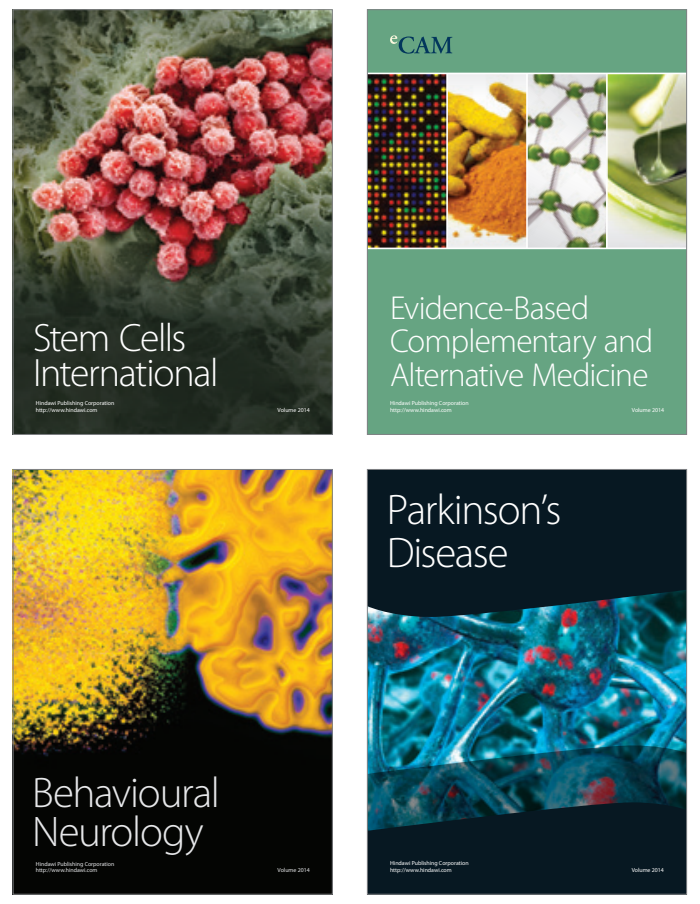
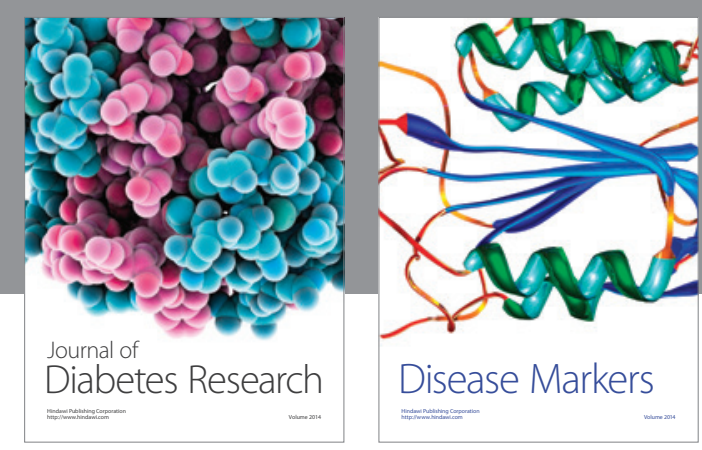

Disease Markers
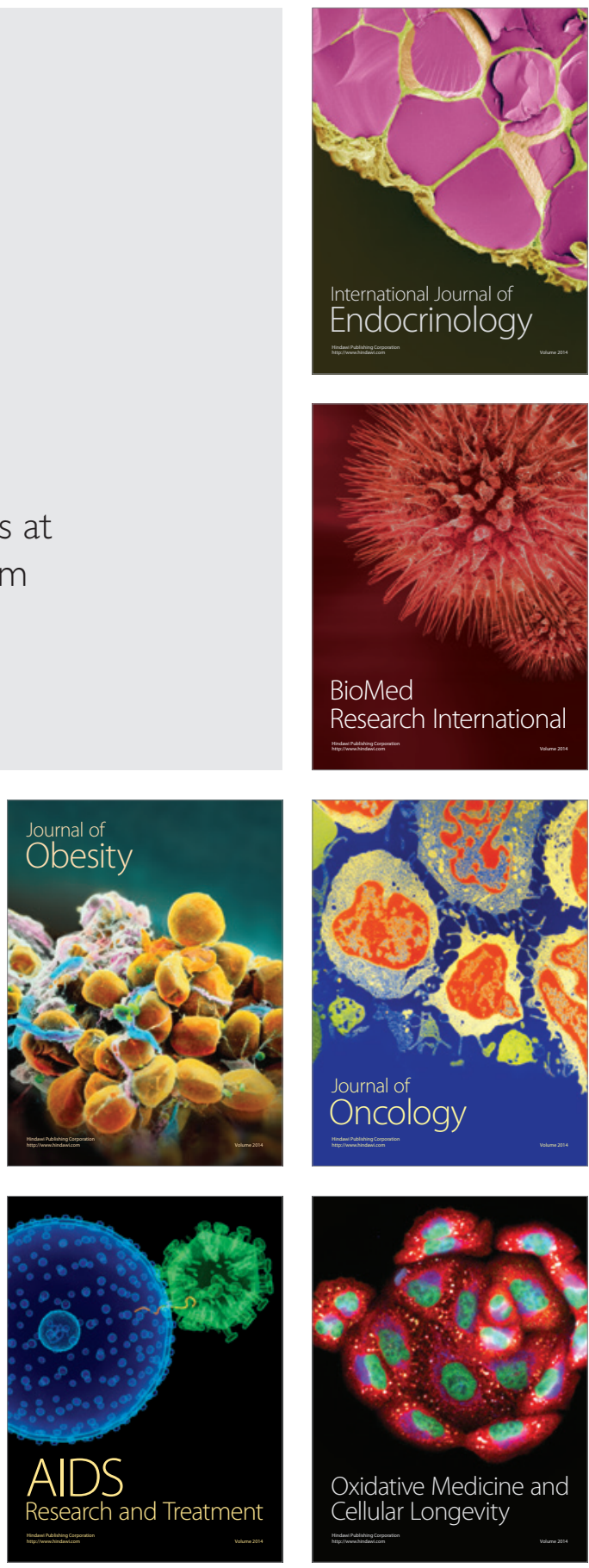